\title{
Regulation of Respiratory and Vocal Motor Pools in the Isolated Brain of Xenopus laevis
}

\author{
Erik Zornik ${ }^{1}$ and Darcy B. Kelley ${ }^{1,2}$ \\ ${ }^{1}$ Department of Biological Sciences and ${ }^{2}$ Program in Neurobiology and Behavior, Columbia University, New York, New York 10027
}

The aquatic frog Xenopus laevis uses a complex vocal repertoire during mating and male-male interactions. Calls are produced without breathing, allowing the frogs to vocalize for long periods underwater. The Xenopus vocal organ, the larynx, is innervated by neurons in cranial motor nucleus (n.) IX-X, which contains both vocal (laryngeal) and respiratory (glottal) motor neurons. The primary descending input to n.IX-X comes from the pretrigeminal nucleus of the dorsal tegmental area of the medulla (DTAM), located in the rostral hindbrain. We wanted to characterize premotor inputs to respiratory and vocal motor neurons and to determine what mechanisms might be involved in regulating two temporally distinct rhythmic behaviors: breathing and calling. Using isolated brain and larynx preparations, we recorded extracellular activity from the laryngeal nerve and muscles and intracellular activity in laryngeal and glottal motor neurons. Spontaneous nerve activities mimicking respiratory and vocal patterns were observed. DTAM projection neurons $\left(D_{T A M}\right.$ IX-X $_{1}$ neurons) provide direct input to glottal and laryngeal motor neurons. Electrical stimulation produced short-latency coordinated activity in the laryngeal nerve. DTAM $\mathrm{IX}_{\mathrm{IX}}$ neurons provide excitatory monosynaptic inputs to laryngeal motor neurons and mixed excitatory and inhibitory inputs to glottal motor neurons. DTAM stimulation also produced a delayed burst of glottal motor neuron activity. Together, our data suggest that neurons in DTAM produce vocal motor output by directly activating laryngeal motor neurons and that DTAM may coordinate vocal and respiratory motor activity.

Key words: hindbrain; parabrachial nucleus; respiration; vocalization; larynx; glottis

\section{Introduction}

The larynx, the vocal organ of many vertebrates, is interposed in the airway between the lungs and the mouth cavity. The glottis serves as a gateway to the larynx; it seals off the lungs from the mouth and prevents the aspiration of food and drink. The glottis must open to permit expiration and inspiration. In most vertebrates, sound production is coupled to expiration both peripherally and within the CNS; the hindbrain circuit that produces communication signals is thought to have evolved from an ancient network that drives breathing (Bass and Baker, 1997). Here we explore the relationship between neural correlates of breathing and calling in an aquatic frog in which (unlike terrestrial vertebrates) these behaviors have lost their obligatory coupling.

Xenopus laevis males produce underwater songs that attract females and establish vocal dominance (Wetzel and Kelley, 1983; Tobias et al., 1998, 2004). Sound production does not require airflow; the contraction of a single laryngeal muscle group causes the snapping apart of paired discs and a resulting click (Yager, 1992), the basic unit of all calls. The simplified mechanism of Xenopus call production makes it an attractive model for address-

Received Dec. 29, 2006; revised Nov. 30, 2007; accepted Nov. 30, 2007.

This work was supported by National Institutes of Health (NIH)-National Research Service Award DC006743 (E.J.Z.) and NIH Grant NS23684 (D.B.K.). We thank Martha Tobias and Sarah Woolley for comments on this manuscript.

Correspondence should be addressed to Dr. Erik Zornik, 5 Cummington Street, Boston University, Boston, MA 02215. E-mail: ez@bu.edu.

D01:10.1523/JNEUROSCI.4754-07.2008

Copyright $\odot 2008$ Society for Neuroscience $\quad$ 0270-6474/08/280612-10\$15.00/0 ing how vocalizations are produced and also offers the opportunity to investigate how breathing and calling can be regulated in a temporally distinct manner.

Previous studies have identified elements of the hindbrain vocal circuit using dye transport methods. Glottal and laryngeal motor neurons (LMNs), detected by their projections to target muscles, are located in cranial nucleus (n.) IX-X (Kelley, 1980; Simpson et al., 1986; Zornik and Kelley, 2007). Nuclei IX-X are coupled bilaterally (Wetzel et al., 1985; Brahic and Kelley, 2003) via commissural interneurons (IX-X $\mathrm{IX}_{\mathrm{IX}}$ neurons) (Zornik and Kelley, 2007). The major descending input to n.IX-X arises from the pretrigeminal nucleus of the dorsal tegmental area of the medulla (DTAM) in the rostral hindbrain (Wetzel et al., 1985; Brahic and Kelley, 2003; Zornik and Kelley, 2007). Based on its location and projections, DTAM is a potential homolog of the mammalian parabrachial nucleus (PB), also known to participate in vocal behavior (Jürgens, 2002; Smotherman et al., 2006). A major neuronal population in DTAM (DTAM ${ }_{\mathrm{IX}-\mathrm{X}}$ neurons) projects to motor neurons throughout n.IX-X. Because of their direct inputs to motor neurons, DTAM $\mathrm{IX}-\mathrm{X}_{\mathrm{X}}$ neurons are excellent focal points for determining how premotor inputs regulate vocalization and respiration.

In vivo recordings from singing frogs have revealed that each click in the calls of $X$. laevis is produced by a coordinated contraction of the laryngeal muscles evoked by a single compound action potential (CAP) in the laryngeal nerve (Yamaguchi and Kelley, 2000). Laryngeal nerve activity in an isolated brain can thus be interpreted with respect to actual vocal behaviors. We used this 

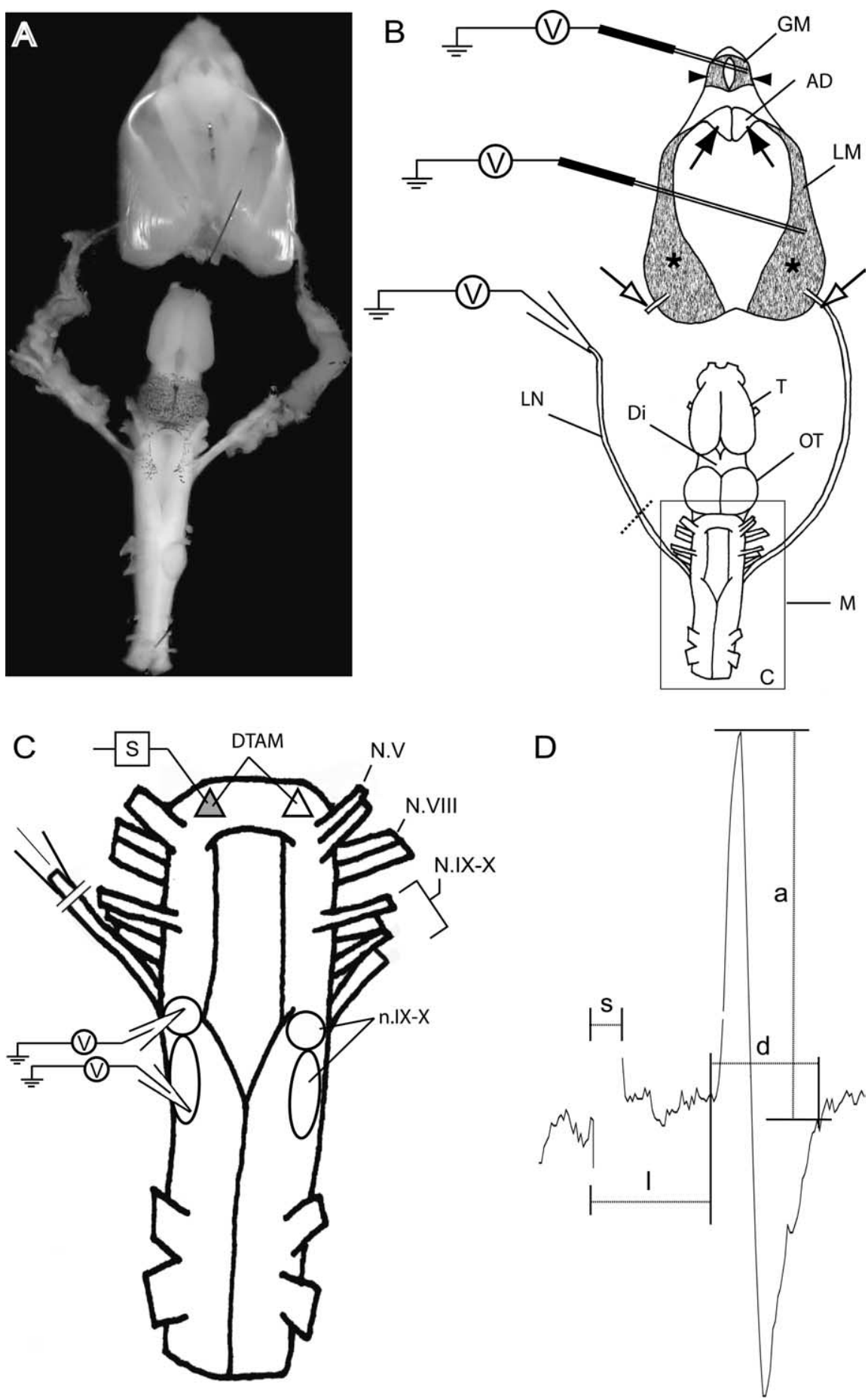

Figure 1. Experimental design: isolated vocal apparatus and physiological recordings. $A$, Photograph of the isolated "intact" vocal preparation: the brain and larynx. The larynx is pinned anterior to the brain (anterior is up). B, Schematic of the vocal apparatus and extracellular electrode configuration. Laryngeal dilator muscles $\left(^{*}\right)$ contract to separate arytenoid cartilage discs (filled arrows). The glottis, anterior to the discs, closed in the relaxed state, is opened by contraction of the glottal muscles on either side (arrowheads). The laryngeal nerve exits the brain via N.IX-X and inserts into the dorsolateral posterior extent of the laryngeal dilators (open arrows). A suction electrode records activity from the cut end of the (left) laryngeal nerve, or the activity of the glottal and laryngeal dilator muscles are recorded directly (right muscles in diagram) when the laryngeal nerve is left intact. C, Detailed diagram of the medulla showing stimulation and intracellular recording sites. Activity in one laryngeal nerve is recorded (left) while the ipsilateral DTAM is stimulated (shaded). Intracellular recordings in n.IX-X were performed ipsilateral to DTAM stimulation sites. D, Example of a typical CAP produced by stimulation. Time between onset of the stimulus artifact $(s)$ and the final upward inflection point before exceeding threshold is measured for CAP latencies (I). CAP duration (d) is measured between onset and the point at which the trace first returns to baseline. Amplitude (a) is measured as maximum deflection from baseline. AD, Arytenoid cartilage disc; Di, diencephalon; GM, glottal muscle; LM, laryngeal dilator muscle; LN, laryngeal nerve; $M$, medulla; 0 T, optic tectum; N.V, trigeminal nerve; N.VIII, auditory nerve; N.IX-X, glossolaryngeal-vagal nerve; T, telencephalon. Brain and larynx diagrams adapted from Zornik and Kelley (2007). preparation to explore the relationship of breathing to calling by recording the activity of laryngeal and glottal motor neurons (extracellular and intracellular recordings) while stimulating DTAM.

\section{Materials and Methods}

Animals and approach. Sexually mature X. laevis males were purchased from Xenopus One (Ann Arbor, MI) and Nasco (Fort Atkinson, WI). Animal care and experimental protocols followed National Institutes of Health guidelines and were approved by the Institutional Animal Care and Use Committee at Columbia University. Two preparations (described below) were used: an isolated brain with the proximal portion of the laryngeal nerve attached to record nerve activity and an isolated brain-larynx preparation in which muscle activity was also recorded. Spontaneous nerve activity and effects of DTAM stimulation were recorded in 109 isolated brain preparations and 26 brain-larynx preparations.

Isolated brain and larynx preparations. The Xenopus larynx, located ventral to the buccal cavity, consists of a cricoid cartilage "box" straddled on either side by the laryngeal dilator muscles (Fig. 1 $A, B$ ). During calling, each muscle contracts simultaneously, rapidly separating two arytenoid cartilage discs (Fig. $1 B$, black arrows); this movement produces a click, the basic unit of all Xenopus calls (Kelley and Tobias, 1999). At the anterior extreme of the larynx lies the glottis, which opens during breathing to allow air to flow from the buccal cavity through the laryngeal cavity and into the lungs. Muscles flank the glottis (closed at rest) and gate the movement of air (Fig. $1 B$, arrowheads). Both laryngeal dilator and glottal muscles are innervated by the laryngeal nerve, which inserts into the dorsolateral-posterior extreme of the laryngeal dilator muscles (Fig. $1 B$, open arrows). The axons that make up the laryngeal nerve exit the brain via the most caudal root of cranial nerve (N.) IX-X along with autonomic efferents (Simpson et al., 1986). To investigate the roles of premotor inputs to laryngeal and glottal motor neurons, we developed three in vitro preparations. To distinguish between glottal and laryngeal motor activities, we dissected the intact "vocal apparatus" in its entirety (Fig. $1 A$ ). This preparation maintains laryngeal nerve connections between the brain and larynx. In most situations, however, a simpler and more rapid dissection was used. The brain and the entire length of one laryngeal nerve was dissected (long nerve configuration), to record nerve activities of only glottal and laryngeal motor pools (Fig. 1B). Finally, once we could distinguish which spontaneous and stimulation-induced activities were laryngeal and which were glottal (as opposed to autonomic efferents, also resident in n.IX-X), we recorded activity in a short nerve configuration, in which $\sim 0.5 \mathrm{~cm}$ of the caudal root of N.IX-X remained intact (Fig. $1 B$, dashed line).

Deeply anesthetized animals $[1.3 \%$ ethyl 3-aminobenzoate methanesulfonate salt 
(Sigma, St. Louis, MO); $1.0 \mathrm{ml}$ injected into the dorsal lymph sac] were placed on ice and transcardially perfused with ice-cold oxygenated saline [in mM: $75 \mathrm{NaCl}, 25 \mathrm{NaHCO}_{3}, 2 \mathrm{CaCl}_{2}, 2 \mathrm{KCl}, 0.5 \mathrm{MgCl}_{2}$, and 11 glucose, oxygenated with carbogen $\left(95 \% \mathrm{O}_{2}, 5 \% \mathrm{CO}_{2}\right)$ to a $\mathrm{pH}$ of 7.3 (from Luksch et al., 1996)] or a modified saline [in mM: $96 \mathrm{NaCl}, 20 \mathrm{NaHCO}_{3}$, $2 \mathrm{CaCl}_{2}, 2 \mathrm{KCl}, 0.5 \mathrm{MgCl}_{2}, 10$ HEPES, and 11 glucose (oxygenated with $99 \% \mathrm{O}_{2}, 1 \% \mathrm{CO}_{2}$ ) to a $\mathrm{pH}$ of 7.6-7.8]. After saline perfusion, the spinal cord was transected caudal to the brachial nerves.

For short nerve preparations, the skull was isolated and placed in freshly oxygenated saline, and the brain was removed maintaining $\sim 0.5$ $\mathrm{cm}$ of the fourth root of N.IX-X. For long nerve preparations, the entire head (along with the underlying buccal cavity and larynx; skin removed) was removed and placed in freshly oxygenated saline. The dorsal surface of the brain was exposed, all nerves except one N.IX-X were cut, and the brain was dissected away from the head, maintaining the nerve connection to the larynx. The laryngeal nerve was then isolated and cut at its muscle insertion point. For EMG recording experiments, the brain and larynx were dissected as for the long nerve preparation, with one or both laryngeal nerves left intact. Extraneous tissue was then removed, allowing us to pin both the brain and larynx (the brain is directly dorsal to the larynx in the intact animal) to a Sylgard-lined (Dow Corning, Midland, MI) recording dish with the larynx anterior to the brain (Fig. $1 A$ ). Tissue was allowed to stabilize in the dish for at least $1 \mathrm{~h}$ before recording began; oxygenated saline was superfused at $>45 \mathrm{ml} / \mathrm{h}$.

Electrophysiological recordings. Activity was recorded from the cut ends of the nerve inserted into tightly fitting suction electrodes fashioned from fire-polished lime glass Pasteur pipettes (VWR International, Batavia, IL) or borosilicate glass capillary tubing (catalog \#30-30-1; Frederick Haer Company, Bowdoinham, ME) drawn on a Flaming/Brown microelectrode puller (model P-87; Sutter Instruments, Novato, CA) and then broken back to fit the nerve. EMG activity was recorded with bipolar differential electrodes, consisting of two Teflon-coated silver wires insulated to the tips (0.007 inch; A-M Systems, Carlsborg, WA), inserted directly into the muscle. Nerve and EMG activities were recorded using a differential alternating current microelectrode amplifier (model 1800; A-M Systems), amplified 1000 times, and bandpass filtered (10 Hz to 1 $\mathrm{kHz}$ ).

DTAM was stimulated using monopolar tungsten electrodes (epoxycoated, $5 \mathrm{M} \Omega$ resistance, $0.005 \mathrm{inch}, 8^{\circ}$ tapered tip; A-M Systems) connected to a stimulator (model S88; Grass Instruments, West Warwick, $\mathrm{RI}$ ) and constant current stimulation isolation unit (PSIU6; Grass Instruments). Meninges dorsal to the nucleus of interest were first removed; external markers were then used to place stimulation electrodes into the correct site. DTAM is located $\sim 1 \mathrm{~mm}$ below the cerebellum immediately caudal to the midbrain/hindbrain border and just lateral to the fourth ventricle (Brahic and Kelley, 2003; Zornik and Kelley, 2007) (for illustration of stimulation site, see Fig. $1 C$ ). Stimulus pulses (300 $\mu$ s in duration) at intensities from 3 to $80 \mu \mathrm{A}$ were applied singly or in trains $(20,40$, and $60 \mathrm{~Hz}$; duration, $1000 \mathrm{~ms})$. Lesions (100-200 $\mu \mathrm{A}, 1 \mathrm{~s})$ were made after some recordings to verify electrode placement in DTAM.

Neurotransmitter antagonists were bath applied. Glutamate receptor antagonists APV and DNQX (dissolved in $0.1 \mathrm{~N} \mathrm{NaOH}$ at high concentrations and diluted to final working concentration in the bath) were used to identify the receptor subtypes underlying DTAM-mediated neurotransmission. Stimulus trains to DTAM were applied before antagonist application to acquire a baseline response. Saline superfusion was then stopped, and the antagonist was added directly to the recording dish. During drug exposure, we continued to record nerve responses to DTAM stimulation at regular intervals (five stimulus sweeps every $2 \mathrm{~min}$ ). After recording these responses, saline superfusion was restarted at $\sim 5 \mathrm{ml} / \mathrm{min}$ to wash out the antagonist, and activities were monitored for $1 \mathrm{~h}$.

To determine whether inhibitory signaling is important in controlling the delayed glottal bursts that followed DTAM stimulus trains, the $\mathrm{GABA}_{\mathrm{A}}$ receptor antagonist bicuculline methiodide (BMI) (Tocris Biosciences, Ellisville, $\mathrm{MO}$ ) was applied to the bath (1 $\mu \mathrm{M}$ final concentration) during DTAM stimulation. DTAM stimulus trains were applied every $30-60 \mathrm{~s}$ for $10 \mathrm{~min}$ before drug application. BMI was then applied, and recordings were continued for $5 \mathrm{~min}$. After each experiment, a high saline flow rate $(\sim 5 \mathrm{ml} / \mathrm{min})$ was used to wash out remaining BMI, and additional recordings confirmed drug recovery. Protocols for control experiments were identical except that saline vehicle was applied to the bath.

For intracellular recordings, meninges along the dorsal hindbrain surface above n.IX-X were removed to allow easy penetration of the recording electrode. High-resistance (40-120 M $\Omega$ ) sharp microelectrodes were made from borosilicate capillary tubing $(1.0 \mathrm{~mm}$ outer diameter $\times 0.75$ $\mathrm{mm}$ inner diameter, with omega dot fiber; Frederick Haer Company) fabricated using a micropipette puller (see above) and filled with a $2 \mathrm{M}$ $\mathrm{KAc}, 20 \mathrm{~mm} \mathrm{KCl}$ solution. In some cases, 2\% Neurobiotin (Vector Laboratories, Burlingame, $\mathrm{CA}$ ) was included in the recording solution. Electrode position was controlled by a Sutter Instruments micromanipulator (MP-285), and recordings were made with an Axoclamp 2 amplifier (Molecular Devices, Sunnyvale CA) in bridge mode. Nucleus IX-X is a column $\sim 1.25 \mathrm{~mm}$ long extending from the nerve insertion to just caudal to the obex. Glottal motor neurons take up $\sim 500 \mu \mathrm{m}$ of the anterior of n.IX-X, and laryngeal motor neurons occupy the posterior $\sim 750 \mu \mathrm{m}$ (Zornik and Kelley, 2007). We therefore targeted glottal motor neurons by inserting the electrode at the level of the nerve insertion point and laryngeal motor neurons by recording at the level of the obex. Electrodes were slowly lowered to $500 \mu \mathrm{m}$ below the brain surface (just above the dorsal extent of n.IX-X) and then advanced in $3 \mu \mathrm{m}$ steps at $50 \mu \mathrm{m} / \mathrm{s}$. Electrode advancement steps were interspersed with capacitance overcompensation "buzz" pulses $(2-20 \mathrm{~ms})$ to penetrate cell membranes. During a cell search, brief negative current pulses ( $50 \mathrm{~ms}$; $-100 \mathrm{pA}$ ) were applied constantly with a $30 \%$ duty cycle; a drop in electrode potential and increased input resistance indicated that a neuron had been impaled. The suction electrode on the nerve ipsilateral to an intracellular electrode was connected to a stimulator and constant-current stimulation isolation unit (see above). Antidromic spikes after 50-100 $\mu \mathrm{A}$ stimulation pulses (300 $\mu$ s duration) positively identified motor neurons.

Data collection. Raw amplified signals were digitized on a Digidata 1322A (Molecular Devices) and monitored using Clampex (PC version), or a MacLab/4s digitizer (AD Instruments, Colorado Springs, CO) interfaced with a Macintosh computer running Chart software (AD Instruments). All data were analyzed with Clampfit (Molecular Devices) and Igor (WaveMetrics, Lake Oswego, OR) software.

Each DTAM stimulus pulse within a train produced a short-latency CAP in the laryngeal nerve. Amplitude, latency, and duration of these responses were quantified in the following manner. A stimulus train (sweep) was repeated five times $(\sim 0.1 \mathrm{~Hz})$ at each rate, and mean values (amplitude and latency) were obtained for stimulus pulses 1-20 (20 data points per trial). Each trial was repeated three times to obtain a final mean value for responses to the first 20 pulses. Root mean squares of noise levels were $\sim 2 \mu \mathrm{V}$; thresholds were set at $5 \mu \mathrm{V}$ in all cases (i.e., at least twice the noise level). CAP amplitudes, defined as the maximum voltage change from baseline, were measured from rectified traces. The final upward inflection point before exceeding threshold was taken as the latency time point (Fig. 1D).

Vocal recordings. Amplectant calls are low intensity, consist of repeated trills of two or three clicks, and are produced by males while clasping another animal. To analyze the interclick intervals of amplectant calling, we primed six sexually mature males and two females with human chorionic gonadotropin (Sigma), which has been shown previously to enhance calling in males and induce sexual receptivity in females (Wetzel and Kelley, 1983; Tobias et al., 2004). Calls produced by male-female pairs were recorded in glass aquaria as described previously (Tobias et al., 2004). Briefly, a mobile hydrophone ( $-52 \mathrm{~dB}, 0.1-6 \mathrm{kHz}$; Knowles, Itasca, IL) attached to a "wand" was used so that the low-intensity calls could be easily detected by holding the hydrophone adjacent to the larynx. At least five amplectant call trills were recorded from each clasping male using a Marantz CD recorder (CDR300; Marantz America, Mahwah, NJ).

Data analysis and statistics. Interclick intervals were measured using GoldWave sound analysis software (GoldWave, St. John's, Newfoundland, Canada). These calls contain either a single interval (for doublets) or a first and second interval (for triplets). Interclick intervals from amplectant calling males were averaged for each animal. The first interval was defined as the interval between doublet clicks or between the first two 
A

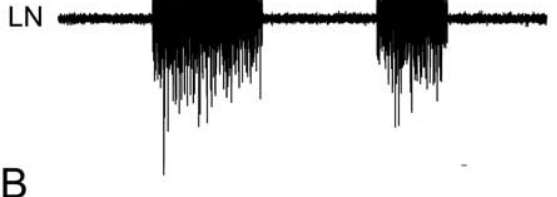

LM

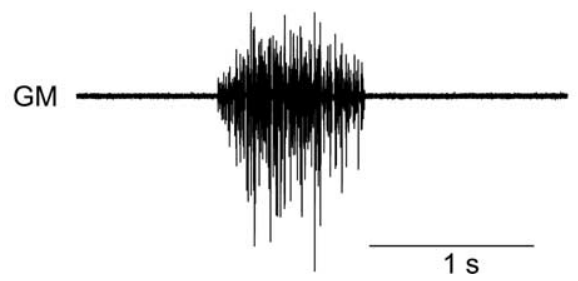

C

$\operatorname{LN}$

N

$\mathrm{D}$

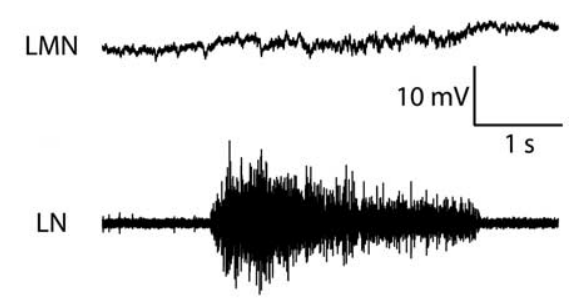

Figure 2. Spontaneous activity in the isolated brain. $A$, Recordings from the $L N$ show spontaneous high-frequency, unpatterned bursts. $\boldsymbol{B}$, In the intact vocal preparation, spontaneous burst activity can be recorded from the glottal (GM), but not laryngeal (LM), muscles. C, Glottal motor neurons (GMN, top trace) rapidly spike during spontaneous glottal bursts on the nerve (bottom trace). D, LMNs (top trace) do not spike during glottal bursts (bottom trace).

clicks in a triplet $(n=6)$. Four of six males produced triplets, so the second intervals (between second and third clicks) were also collected and averaged. To compare the nerve CAPs to the vocal interclick intervals, we analyzed inter-CAP intervals (from six randomly selected preparations) and performed a one-way ANOVA with Tukey's post hoc comparisons between the first and second intervals.

In addition to latency, two additional values were measured: CAP onset "jitter" and CAP duration. These are useful measurements for identifying monosynaptic connections, because they indicate the relative synchrony of motor neuron firing. Jitter values (defined as the SD of the mean CAP onset latency) were used in cross-experiment comparisons. CAPs progressively increase in amplitude within a stimulus train; to more accurately detect onset latencies, we calculated CAP onset values for stimulus pulses 11-20 in which responses are reliably larger. One jitter value was generated for each stimulus rate from 100 or 150 onset latencies (10 pulses per sweep, five sweeps per trial, two or three trials). CAP onset latency and jitter values for each rate were compared using a one-way ANOVA with Tukey's post hoc tests (for comparing results at different stimulus rates). EPSP latency and jitter were calculated for three neurons based on time to half-peak amplitude (measured from pulses 11-20 of $20 \mathrm{~Hz}$ stimulus trains). CAP durations were measured from nonrectified traces (Fig. 1D) by using the onset time (as for latency measurements) and the point at which the trace returns to baseline. We used a repeated-measures ANOVA and Tukey's multiple comparison post hoc tests to compare mean CAP durations between 20,40 , and $60 \mathrm{~Hz}$ rates of stimulation.

A two-way repeated-measures ANOVA was used to compare effects of APV and DNQX before and after treatment; Bonferroni's post hoc tests were performed to compare across antagonists. Because poststimulus burst latencies were variable, values from BMI or control conditions were normalized to a baseline value (average of at least three pretreatment burst latencies), and experimental conditions were compared as percentage control using a two-tailed $t$ test.

Figures of physiological traces were prepared using Igor (WaveMetrics), and graphs were generated using Microsoft (Seattle, WA) Excel. Prism (GraphPad Software, San Diego, CA) was used for statistical analyses. Unless otherwise noted, all data are reported as mean \pm SE.

Histology. After a stimulation site was marked with a lesion, brains were fixed overnight in $4 \%$ paraformaldehyde in $0.1 \mathrm{M}$ phosphate buffer, cryoprotected in $20 \%$ sucrose, and then sectioned horizontally $(30 \mu \mathrm{m})$ on a cryostat (Hacker Instrument, Fairfield, NJ). Sections were allowed to dry on slides for at least $4 \mathrm{~h}$ and then stained with cresyl violet, dehydrated, cleared in xylene, and coverslipped. To detect Neurobiotin fills, dried cryostat sections were processed using Vectastain Universal $\mathrm{ABC}$ and $\mathrm{DAB}$ substrate kits (Vector Laboratories) and then counterstained as above. Bright-field photomicrographs were obtained on a Leica (Nussloch, Germany) DMR microscope with a digital Spot Camera (software version 4.0.4; Diagnostic Instruments, Sterling Heights, MI). Adobe Photoshop (Adobe Systems, San Jose, CA) was used to correct unevenness of illumination (using the dodge tool) and to enhance tissue outline and cellular details (using the levels function).

\section{Results}

Nerve recordings: spontaneous activity Spontaneous activity was recorded from some perfused isolated brains; the most common pattern recorded from the laryngeal nerve is a high-frequency unpatterned burst (Fig. 2A). Spontaneous bursts were observed in approximately one-third of preparations; approximately two-thirds of brains that appear electrophysiologically robust (i.e., electrical stimulation produced typical nerve responses; see below) do not display bursting. When bursts are recorded, durations range from 218 to $3508 \mathrm{~ms}$ (average of $1103 \pm 113 \mathrm{~ms} ; n=30$ preparations). Bursts can occur in bouts as fast as $0.5 \mathrm{~Hz}$ but are usually less frequent, approximately once or twice per minute $(1.7 \pm 0.3$ per $\min , n=13$ preparations; calculated from experiments with at least $3 \mathrm{~min}$ of spontaneous burst recordings).

The laryngeal nerve, as it enters the dilator muscles, contains axons of two populations of motor neurons: laryngeal and glottal. To determine the origin of the unpatterned bursts, we recorded EMGs from glottal and laryngeal muscles in the intact brainlarynx preparation. Spontaneous, unpatterned EMG bursts were recorded from glottal, but not laryngeal, muscles (Fig. 2 B). We observed glottal muscle activity coincident with glottal opening. We thus conclude that the high-frequency, unpatterned bursts of activity recorded from the laryngeal nerve leads to glottal opening. Because the glottal muscles do not contract myogenically, these high-frequency bursts represent the activity of glottal motor neurons.

To target glottal motor neurons for intracellular recording, we directed the electrode to anterior n.IX-X as glottal motor neurons predominate there (Zornik and Kelley, 2007). Intracellular recordings from antidromically identified motor neurons in anterior n.IX-X reveal spiking activity that coincides with unpatterned bursts recorded on the laryngeal nerve (Fig. 2C). Spontaneous bursts occurred during three of five glottal motor neuron recordings; in the other two neurons, bursts were induced by DTAM stimulation. For two neurons, intracellular fills verified location in anterior n.IX-X. Intracellular recordings from antidromically identified motor neurons in posterior n.IX-X revealed that these cells did not spike during unpatterned laryngeal bursts (Fig. 2D). We conclude that the unpatterned bursts (recorded in the laryngeal nerve and glottal muscle) are driven by spiking activity in glottal motor neurons in anterior n.IX-X. The posterior motor neurons represent the second motor pool in n.IX-X (Simpson et al., 1986), laryngeal motor neurons.

A second activity pattern recorded from the laryngeal nerves is doublet or triplet CAPs (Fig. 3). CAP intervals resemble the interclick intervals of amplectant calls: doublet and triplet click 

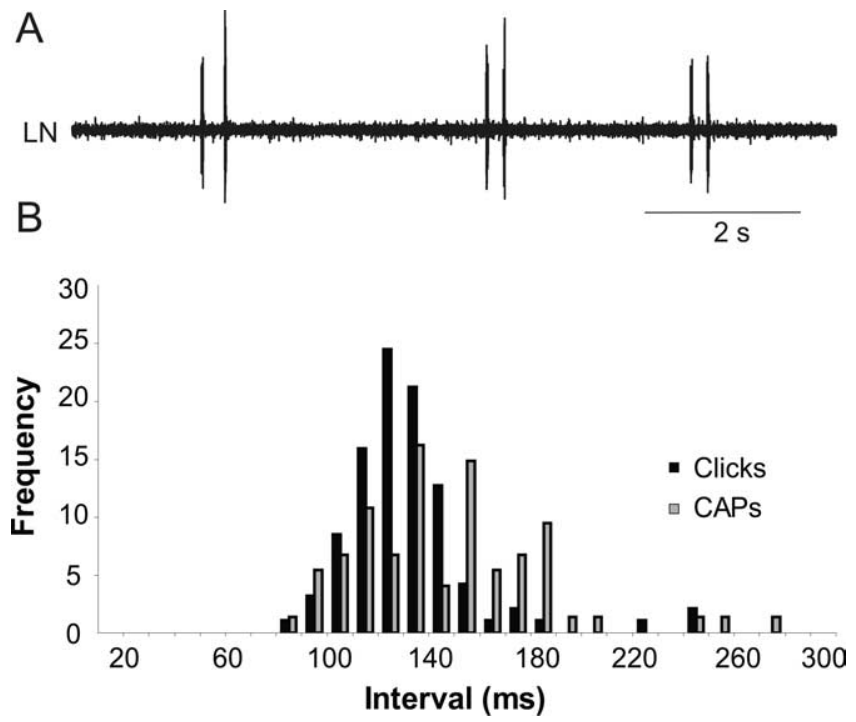

Figure 3. A, Doublet CAPs (resembling the Xenopus amplectant call pattern) are produced spontaneously by the isolated brain. $\boldsymbol{B}$, Histogram showing the distribution of amplectant call click intervals ( $n=6$ animals) and spontaneous CAP intervals ( $n=6$ preparations) (data are only presented for the first interval; see Results).

trills produced by males clasping a female or another male (Kelley and Tobias, 1999). Of the 30 preparations that produced unpatterned glottal bursts, eight also produced doublet and triplet CAPs; one preparation that did not produce spontaneous glottal bursts did produce doublet and triplet CAPs. Occasionally, the two activity patterns were present during a single recording; a likely interpretation of such a recording is that it represents alternating activity of glottal and laryngeal motor neurons. Mean CAP duration was $10.0 \pm 1.6 \mathrm{~ms}$. The inter-CAP intervals were analyzed from six randomly selected preparations that displayed this activity pattern. The mean CAP interval is $144 \pm 9.1 \mathrm{~ms}(n=6)$ between the first two CAPs. Interclick times for the first interval of actual calls (mean $138 \pm 10 \mathrm{~ms}, n=6$ animals) are not statistically different from the CAP intervals (Fig. $3 B)(p>0.05, n=6)$. The interclick times of the second interval, however, were shorter in singing frogs $(141 \pm 7 \mathrm{~ms})$ than the inter-CAP intervals produced by the isolated brain $(232 \pm 38 \mathrm{~ms})(p<0.05$, $n=4)$.

\section{Nerve recordings: DTAM stimulation-induced CAPs}

DTAM (Fig. $4 B$ ) is a $\sim 300-\mu \mathrm{m}$-diameter nucleus in the rostral hindbrain, ventral to the cerebellum. Electrical stimulation of DTAM (electrode tips placed within the nucleus) produced pronounced and highly stereotyped CAPs (Fig. 4A), verified by postrecording lesions (Fig. 4B) (see Materials and Methods). The mean CAP durations were brief and did not vary with stimulation rate $(3.5 \pm 0.2,3.7 \pm 0.1$, and $3.6 \pm 0.2$ for 20,40 , and $60 \mathrm{~Hz}$, respectively; $p>0.05$ for all comparisons, $n=3$ ) suggesting a high degree of synchrony.

Stimulating DTAM with trains at 20, 40, and $60 \mathrm{~Hz}$ produced one-to-one CAPs in the laryngeal nerve (Fig. 4C-E). CAP amplitudes increased within a train at each rate tested: $20 \mathrm{~Hz}, 51 \pm 15$ $\mu \mathrm{V} ; 40 \mathrm{~Hz}, 63 \pm 28 \mu \mathrm{V} ; 60 \mathrm{~Hz} 26 \pm 16 \mu \mathrm{V}$ (CAP amplitudes produced by stimulus pulse 20 compared with baseline CAP amplitude of $\sim 18 \mu \mathrm{V}$ ) (Fig. $4 F$ ).

During the first stimulus pulses in a train, CAP amplitudes increase (Fig. 4) and latencies decrease (Fig. $5 \mathrm{~A}$ shows an example of average CAP latencies across $20 \mathrm{~Hz}$ DTAM stimulus trains in a single preparation). Latencies were similar for 20,40 , and $60 \mathrm{~Hz}$ stimulations $(7.0 \pm 0.6,7.0 \pm 0.9$, and $7.3 \pm 0.7 \mathrm{~ms}$, respectively; $p>0.05$ for each comparison, $n=3)$. Jitter values were small and did not vary with stimulation rate $(251 \pm 8,208 \pm 24$, and $289 \pm$ $88 \mu$ s, for 20,40 , and $60 \mathrm{~Hz}$ respectively; $p>0.05$ for all comparisons, $n=3$ ) (Fig. $5 B$ ).

The small jitter values for DTAM stimulation-induced CAPs suggest a monosynaptic connection. To examine this possibility, we recorded intracellularly from six laryngeal motor neurons (identified as described above). The mean EPSP latency for three representative neurons was $6.5,7.9$, and $7.3 \mathrm{~ms}$ (Fig. 5C). The within-neuron SD (jitter) is small: 135, 540, and $220 \mu$ s, respectively. The DTAM to LMN synapse has very high fidelity, with a $0 \%$ failure rate (every DTAM stimulus pulse resulted in either one EPSP or one spike). This observation also supports the interpretation that the connection between DTAM and laryngeal motor neurons is monosynaptic.

Nerve and muscle recordings after stimulation: identification of a presynaptic neurotransmitter and the postsynaptic motor neuron

The AMPA and NMDA glutamate receptor antagonists (DNQX and APV, respectively) were used to determine whether the DTAM inputs to n.IX-X were glutamatergic. Nerve activity was reversibly blocked by DNQX (10 $\mu \mathrm{M})$ (Fig. 6A). CAP amplitudes decreased within 1-2 min, and CAPs were completely eliminated (Fig. 6A) $15 \mathrm{~min}$ after application; this effect was highly significant $(p<0.001)$. The effects of DNQX were partially reversible but required a washout period in excess of $1 \mathrm{~h}$ (Fig. 6C). Neither APV $(50 \mu \mathrm{M})$ (Fig. $6 B)$ nor a low dose $(1 \mu \mathrm{M})$ of DNQX decreased CAP amplitudes $(p>0.05$ in both cases; $n=5$ and 3 , respectively).

Does DTAM-induced nerve activity represent the activity of laryngeal or glottal motor neurons? We recorded EMG potentials from laryngeal dilator and glottal muscles from the intact brain-larynx preparation while stimulating DTAM (26 preparations). Recordings during DTAM stimulation show that EMGs follow stimuli one-to-one in laryngeal muscle but not glottal muscle (Fig. 7A). DTAM stimulation produced EMG potentials that were similar in ipsilateral and contralateral muscles (data not shown), supporting the anatomical finding of a strong bilateral projection from DTAM to n.IX-X (Zornik and Kelley, 2007).

\section{Intracellular recordings after DTAM stimulation}

We recorded intracellularly from five glottal motor neurons. DTAM stimulation produced hyperpolarization (Fig. 7B). Closer examination (Fig. 7B, expanded) reveals a short-latency EPSP truncated by an IPSP. Occasionally, the short-latency EPSP resulted in an action potential (Fig. 7C). Glottal motor neurons thus receive both excitatory and inhibitory inputs. Consonant with this finding, DTAM stimulus trains delivered during spontaneous glottal bursts result in a brief pause in nerve activity after each pulse (Fig. 7D).

We also recorded intracellular activity of six laryngeal motor neurons in posterior n.IX-X. Each stimulus pulse delivered to DTAM produced an EPSP or single spike in laryngeal motor neurons (Fig. 7E, top trace); each response coincided with a CAP recorded from the laryngeal nerve (Fig. $7 E$, bottom trace). This one-to-one pattern was maintained from 20 to $60 \mathrm{~Hz}$ (Fig. 7F). 
A
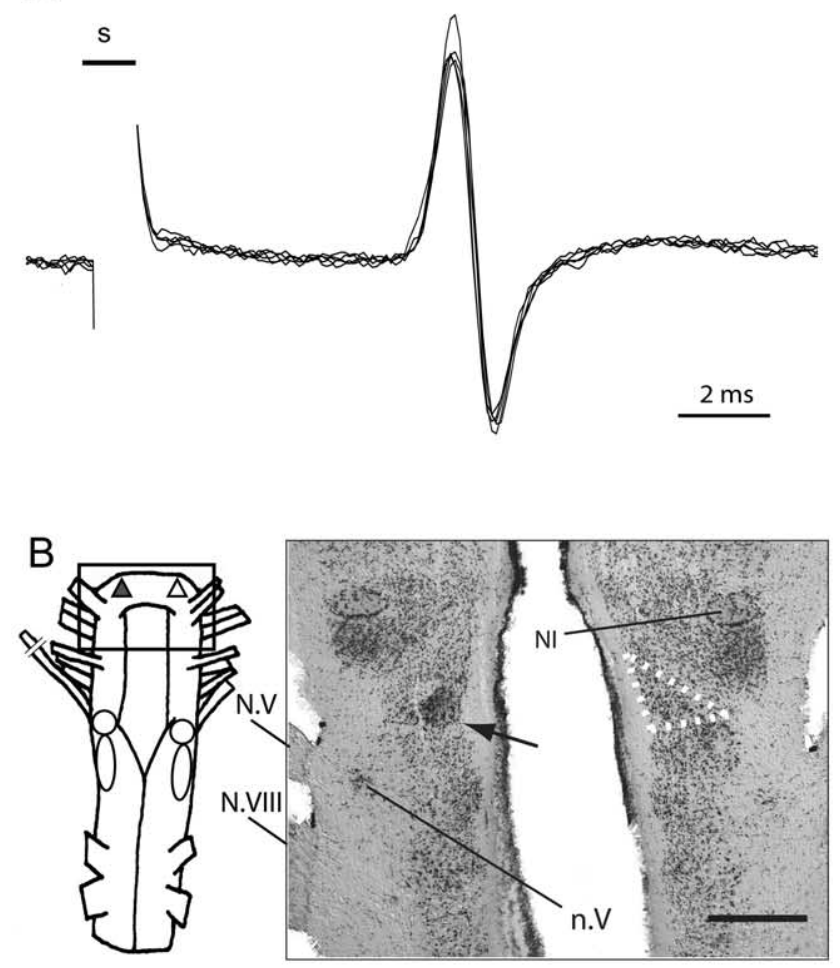

C
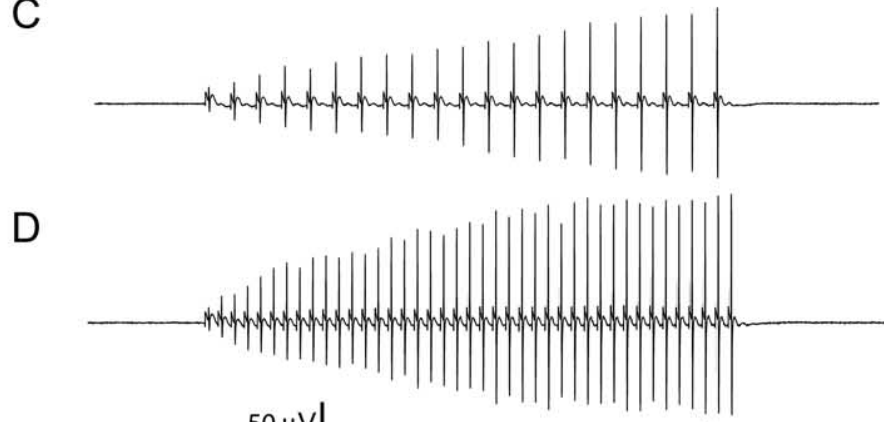

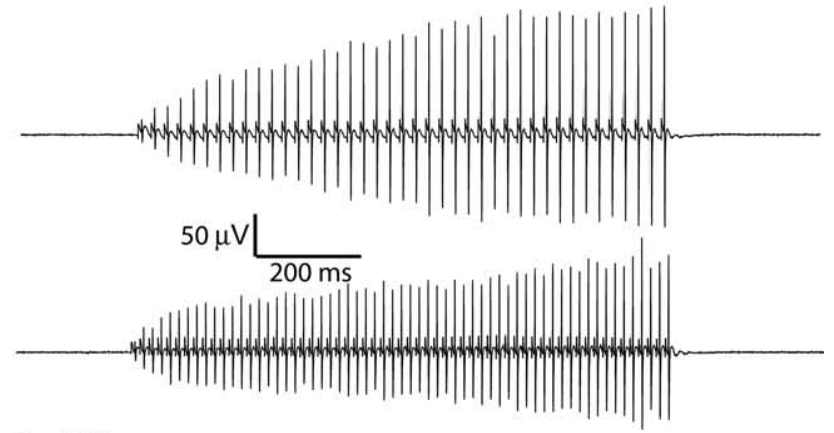

$\mathrm{F}$

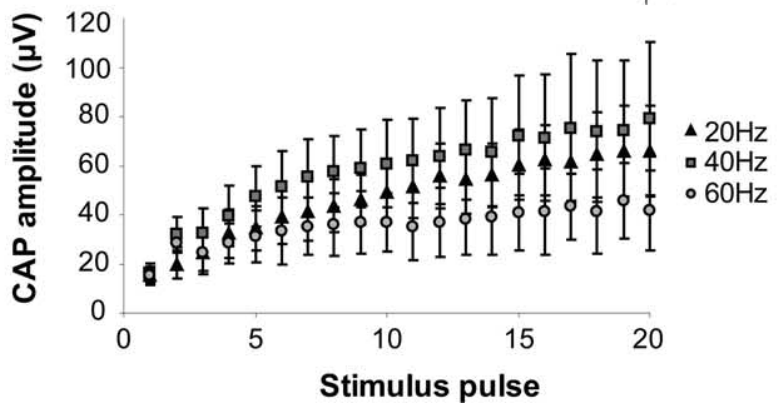

Figure 4. DTAM stimulation: nerve recordings. $A$, Repeated DTAM stimulation produces coordinated, stereotyped CAPs (5 overlaid traces are shown). B, Diagram of the medulla; shaded triangle outlines DTAM stimulation site. Box indicates area shown in adjacent photomicrograph. Nissl-stained horizontal section shows lesion (arrow) centered in the left DTAM. DTAM (outlined on the right side) is medial and caudal to nucleus isthmi (NI) and anteromedial to the motor nucleus of the trigeminal (n.V). C, DTAM stimulation at $20 \mathrm{~Hz}$ produces a potentiating series of CAPs. D, E, The $40 \mathrm{~Hz}$ (D) and $60 \mathrm{~Hz}(\boldsymbol{E})$ stimulation also elicits amplitude modulated CAPs. $\boldsymbol{F}$, Means of first 20 CAPs during DTAM stimulus trains (quantification of 3 representative preparations). The $40 \mathrm{~Hz}$ trains elicit the greatest increase in CAP size; stimuli at $60 \mathrm{~Hz}$ elicit the least amount of amplitude modulation. Traces represent an average of five stimulus sweeps.

\section{DTAM stimulation: relationship of laryngeal activity to glottal bursts}

DTAM stimulation also produced posttrain bursts in the laryngeal nerve (Fig. 8A) that closely resemble spontaneous glottal bursts (Fig. 2). Burst latencies ranged from 276 to $4307 \mathrm{~ms}$; the mean burst duration, $1269 \pm 220 \mathrm{~ms}$ ( $n=18$ preparations), was not significantly different from burst durations of spontaneous glottal bursts ( $p=0.46$, unpaired $t$ test, two-tailed). DTAMinduced bursts represent the activity of glottal motor neurons; in the brain-larynx preparation, poststimulus EMG potentials were recorded from glottal, but not laryngeal, muscles (Fig. $8 \mathrm{~B}$ ) and were accompanied by opening of the glottis. Intracellular recordings also confirmed that glottal motor neurons fired bursts of action potentials during a poststimulus burst (data not shown). The burst delay in response to DTAM stimulation depends on GABAergic transmission. Control experiments (perfusion stopped, saline vehicle added to bath) showed some reduction in poststimulus burst latency $(47 \pm 16 \% ; n=4)$; however, bath application of $1 \mu \mathrm{M}$ bicuculline (Fig. $8 \mathrm{C}$ ) produced a significantly greater reduction in latency $(92 \pm 6 \% ; p=0.02$, two-tailed $t$ test; $n=5)$. Actual mean latencies were much shorter $(93 \pm 73 \mathrm{~ms})$ during bicuculline treatment compared with control conditions $(747 \pm 177 \mathrm{~ms})$.

\section{Discussion}

Identification of neuron types in the hindbrain vocal circuit The main elements of the hindbrain vocal circuit have been outlined previously in the isolated brain using transport of dyes injected into laryngeal and glottal muscles, n.IX-X and DTAM
(Zornik and Kelley, 2007). These studies defined two motor neuron pools in n.IX-X (glottal and laryngeal) and two classes of interneurons (commissural and DTAM projecting) (Fig. 9). Neurons in DTAM project to all four of these n.IX-X cell types, and these inputs appear to be robust. For example, the DTAM to LMN projection ensheathes the postsynaptic neuron soma and terminals. What cannot be determined from dye transport, however, is the valence of the projection (excitatory or inhibitory), its neurotransmitter, and its strength. In addition, only direct projections were seen (the dyes did not appear to cross synapses).

Extracellular recording from the laryngeal nerve, intracellular recordings from motor neurons, and stimulating DTAM in the presence and absence of neurotransmitter blockade has provided a more complete picture of hindbrain vocal circuitry (Fig. 9). The input from DTAM to LMNs is excitatory and strong. Intracellular recordings reveal no synaptic failures and short synaptic delays. Low jitter values for CAPs and EPSPs are similar to monosynaptic connections in other systems (Markram et al., 1997; Doyle et al., 2002; Feldmeyer et al., 2002; Wierenga and Wadman, 2003). The high degree of motor neuron synchrony revealed by short CAP durations also suggests a monosynaptic connection. Because DTAM stimulation-induced CAPs are abolished by blocking glutamate receptors, we conclude that DTAM neurons project to laryngeal motor neurons monosynaptically using glutamate as an excitatory neurotransmitter; pharmacological evidence supports action via the AMPA class of receptors. The timing of clicks within the male advertisement call is highly stereotyped (Kelley and Tobias, 1999; Rhodes et al., 2007), a characteristic matched 

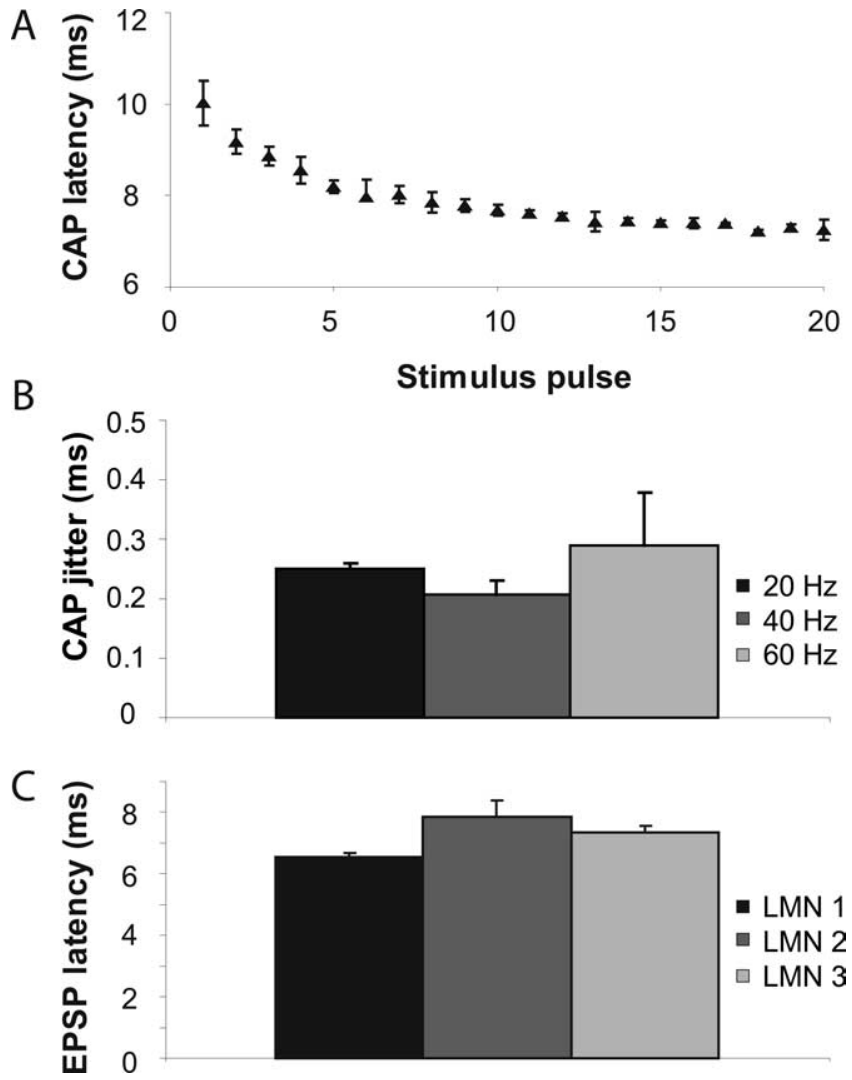

Figure 5. DTAM stimulation: CAP and EPSP latency and jitter. $A$, Average CAP onset latencies during DTAM stimulation $(20 \mathrm{~Hz})$ in one representative brain; latencies decrease as stimulus trains progress. $\boldsymbol{B}$, CAP jitter values are small and do not vary across DTAM stimulus rates. $\boldsymbol{C}$ DTAM-induced EPSPs in LMNs have latencies from 6 to $8 \mathrm{~ms}$. Jitter values (SD of the mean; error bars) are small.

by the precision of DTAM-induced CAPs. The nature of the DTAM to LMN connection may be essential for producing this highly robust behavior.

Intracellular recordings reveal that glottal motor neurons also receive excitatory input from DTAM. Unlike laryngeal motor neurons, however, DTAM stimulation does not produce stimulus-locked spikes concomitant with CAPs in the laryngeal nerve; glottal bursts are evoked by DTAM stimulation but lag the activity of laryngeal motor neurons. This lag is abolished when GABAergic transmission is blocked. The most likely explanation for this finding is that DTAM stimulation activates inhibitory interneurons that project to glottal, but not laryngeal, motor neurons (Fig. 9). This interpretation is supported by DTAM-evoked IPSPs in glottal motor neurons and by the pauses in spontaneous glottal bursting that occur in response to DTAM stimulation. We do not know where these inhibitory interneurons are located. DTAM could contain these inhibitory interneurons or activate inhibitory interneurons in its target, n. IX-X; both include GADpositive neurons (Hollis and Boyd, 2005).

Does LN activity represent fictive breathing and/or calling? $X$. laevis communicate using underwater trills whose communicative significance is encoded by temporal pattern and rate (Vignal and Kelley, 2007). These features are conveyed from the brain to the vocal organ via the laryngeal nerve, in which activity patterns precisely correspond to those of the calls themselves (Yamaguchi and Kelley, 2000). The perfused, isolated brain spontaneously produces activity patterns that closely match a call
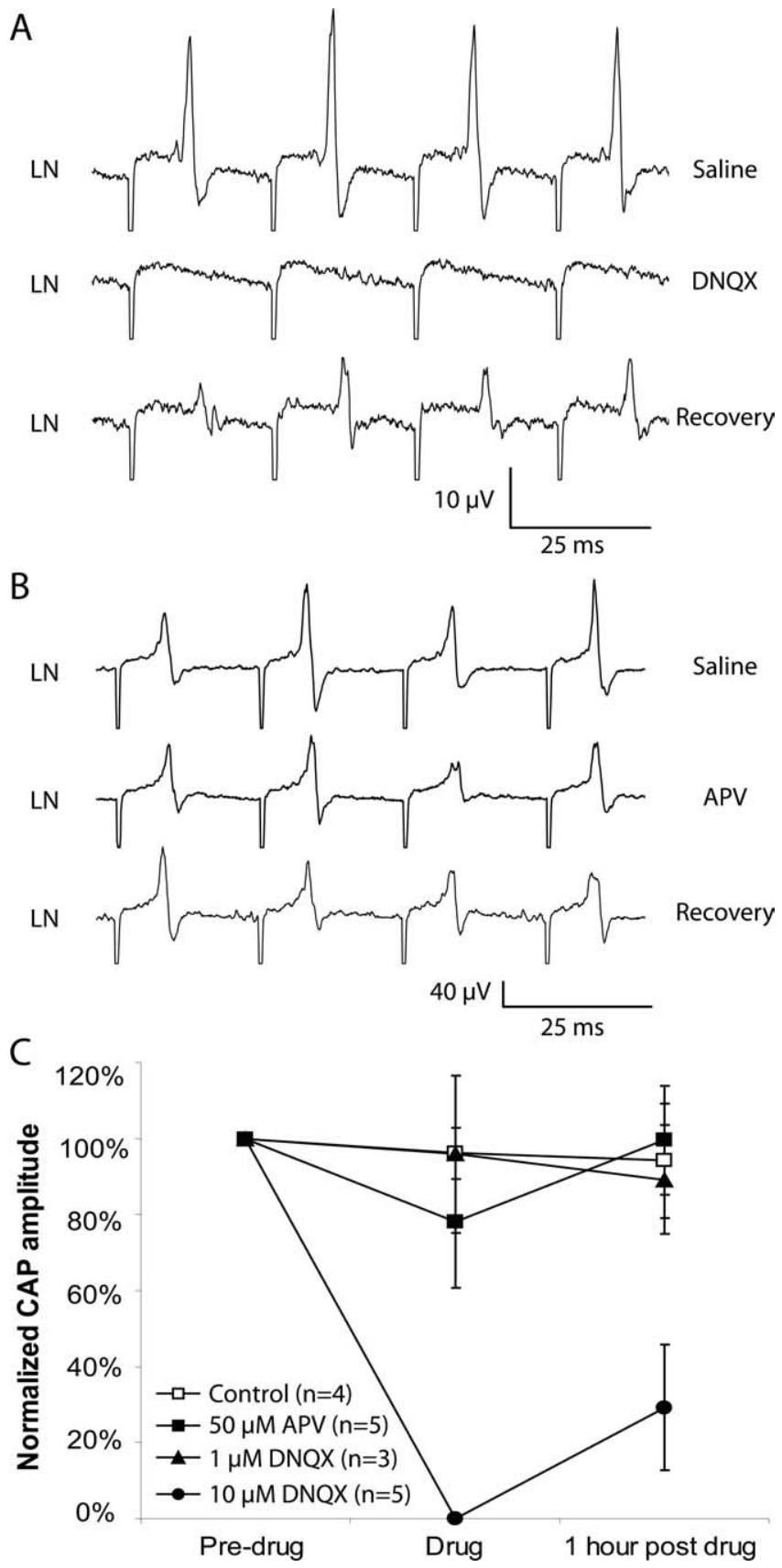

Figure 6. Premotor stimulation: identification of the presynaptic transmitter. $\boldsymbol{A}$, Shortlatency CAPs produced in response to DTAM stimulation (top trace) are eliminated in the presence of $10 \mu \mathrm{m}$ bath-applied DNQX (middle trace). Recovery can be achieved after $>1 \mathrm{~h}$ washout (bottom trace). B, DTAM-induced CAPs (top trace) are not reduced by bath application of $50 \mu \mathrm{M}$ APV (middle trace). C, Chart summarizing effects of APV and DNQX application to DTAM stimulation-induced CAPs. Values are normalized to pretreatment amplitudes (left column) and expressed as percentage of that value during drug application (middle column) and after washout (right column). All five $10 \mu \mathrm{m}$ DNQX trials completely eliminated CAP production (black circle, center). LN, Laryngeal nerve.

made by male frogs when clasping: the amplectant call. This call consists of doublet or triplet clicks. The interclick intervals for doublets or the first interval of triplets cannot be discriminated from inter-CAP intervals recorded from the laryngeal nerve. We conclude that these doublet and triplet CAPs are neural correlates of amplectant calling.

The perfused, isolated brain also produces a second spontaneous activity pattern: high-frequency, unpatterned bursts (this 
A
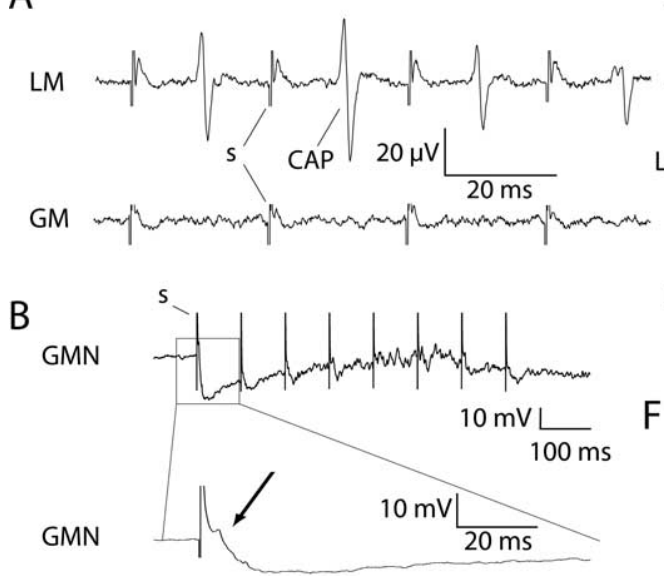

C

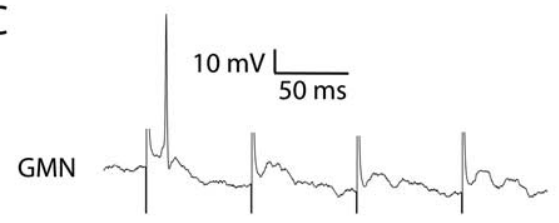

D

LN

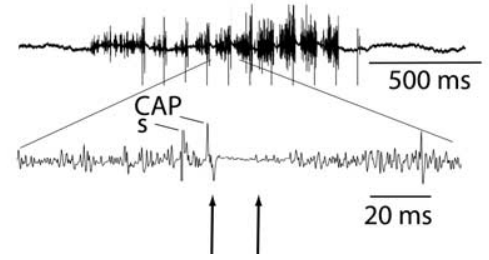

$E$

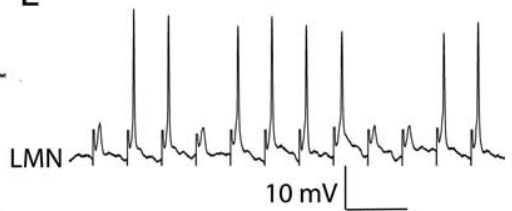

LN

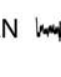

$\mathrm{F}$

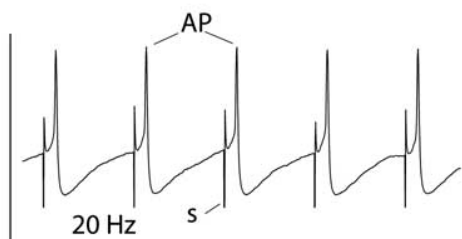

LMN

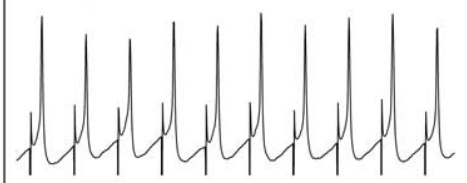

$40 \mathrm{~Hz}$

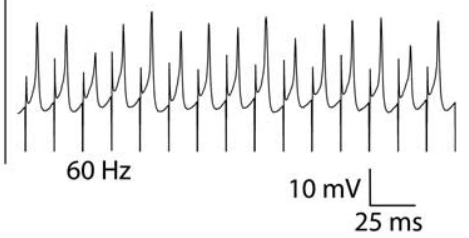

Figure 7. Distinct inputs from DTAM to laryngeal and glottal motor neurons. $\boldsymbol{A}$, Recordings from laryngeal dilator muscle (LM) and glottal muscle (GM) during DTAM stimulation show EMG potentials recorded from laryngeal dilator muscle but not glottal muscle, suggesting that DTAM-induced CAPs in the nerve represent laryngeal motor neuron activity. $\boldsymbol{B}$, During stimulation of DTAM, glottal motor neurons (GMNs) are inhibited (top trace) but receive mixed excitatory and inhibitory inputs. The bottom, expanded trace shows an example of an EPSP (arrow) truncated by inhibitory input. $\boldsymbol{C}$, In some cases, glottal motor neuron EPSPs produced by DTAM stimulation lead to a spike, usually at the beginning of a train. $\boldsymbol{D}$, Stimulating DTAM during a spontaneous glottal burst results in brief pauses in activity after each stimulus pulse (top trace). Enlarged view of a single DTAM stimulation pulse (bottom trace) shows a resulting CAP and the subsequent pause in glottal burst activity (arrows). $\boldsymbol{E}$, Intracellular recordings from LMNs (top trace) during DTAM stimulation shows a single EPSP or spike after each pulse, coming shortly before a CAP in the nerve (bottom trace). $\boldsymbol{F}$, One-to-one action potentials (AP) are induced by 20,40 , and $60 \mathrm{~Hz}$ DTAM stimulation.

pattern has also been reported by Rhodes et al., 2007). These bursts closely resemble those recorded from glottal muscles during opening and coincide with intracellular activity recorded from glottal motor neurons. The high-frequency, unpatterned bursts thus represent the activity of glottal motor neurons in n.IX-X. The glottis must be open to breathe. During breathing in intact Xenopus, high-frequency, unpatterned bursts can be recorded from the laryngeal nerve (Rhodes et al., 2007). Thus, glottal motor neuron bursts are neural correlates of breathing.

Although both activity patterns, fictive amplectant calling and fictive breathing, can be recorded from the laryngeal nerve in the same preparation, they have not been observed simultaneously (either spontaneously or in response to DTAM stimulation). We conclude that the $X$. laevis isolated brain preparation can spontaneously produce both respiratory and vocal patterns, and the temporal segregation of breathing and calling observed in vivo appears to be maintained in vitro.

\section{Generating vocal patterns}

Two results of DTAM stimulation have been reported. The first, observed both here and in another study (Rhodes et al., 2007), is a 1:1 relation between nerve CAPs and DTAM stimulation. A second effect of DTAM stimulation in that study (in two of eight preparations) is fictive advertisement calling with a variable latency between stimulation and nerve activity. We only observed advertisement calling correlates in the perfused brain once; the most likely contributors to this difference in results are site, duration, and intensity of stimulation.

In male $X$. laevis brains, application of serotonin produces patterned activity in the laryngeal nerve that closely resembles advertisement calling (Rhodes et al., 2007). Fictive advertisement calling induced by 5-HT application requires an intact connection between DTAM and n.IX-X; DTAM thus plays an essential role in generating calls. With respect to respiration, we find that DTAM stimulation can evoke glottal bursting (rapidly if inhibition is attenuated). DTAM-lesioned, isolated brains continue to produce glottal bursting (Rhodes et al., 2007); thus the DTAM to n.IX-X projection is not required for generating the neural activity pattern associated with breathing. This connection, however, does appear to prevent glottal activity while laryngeal motor neurons are active and may be essential for coordinating breathing and calling by regulating the onset or offset of respiration. Rostral hindbrain control of respiratory production is consistent with the hypothesis that DTAM is a homolog of the mammalian parabrachial nucleus (see below).

\section{The coordination of breathing and calling in vertebrates}

In most terrestrial vertebrates, vocalization and respiration are tightly coupled; sound production can be gated by expiration (and the timing of inspiratory onset) and the relevant motor neurons share afferents. In mammals, in which respiration has been intensively studied, a group of nuclei in the pons, the parabrachial complex or PB, is known to participate in both vocalization and respiration. Spanning the midbrain/hindbrain tegmentum, the PB has been identified as a key component of the mammalian vocal circuit based on its projections to both laryngeal and respiratory nuclei (Jürgens, 2002). In bats, modifying GABAergic transmission in the lateral PB alters vocalization durations by altering the duration of expiration (Smotherman et al., 2006). The location of DTAM and its projections to vocal and respiratory motor neurons, as well as other interneurons in n.IX-X, support a possible homology with the PB. However, unlike mammals (and terrestrial frogs), in which expiration is required for vocalization, in Xenopus breathing and calling are mutually exclusive.

\section{Respiration and vocalization: evolutionary considerations}

Bass and Baker (1997) have proposed that vocal circuits arose from other, more ancient, rhythmic networks in the caudal hindbrain. Primary among these is the respiratory circuit, to which 


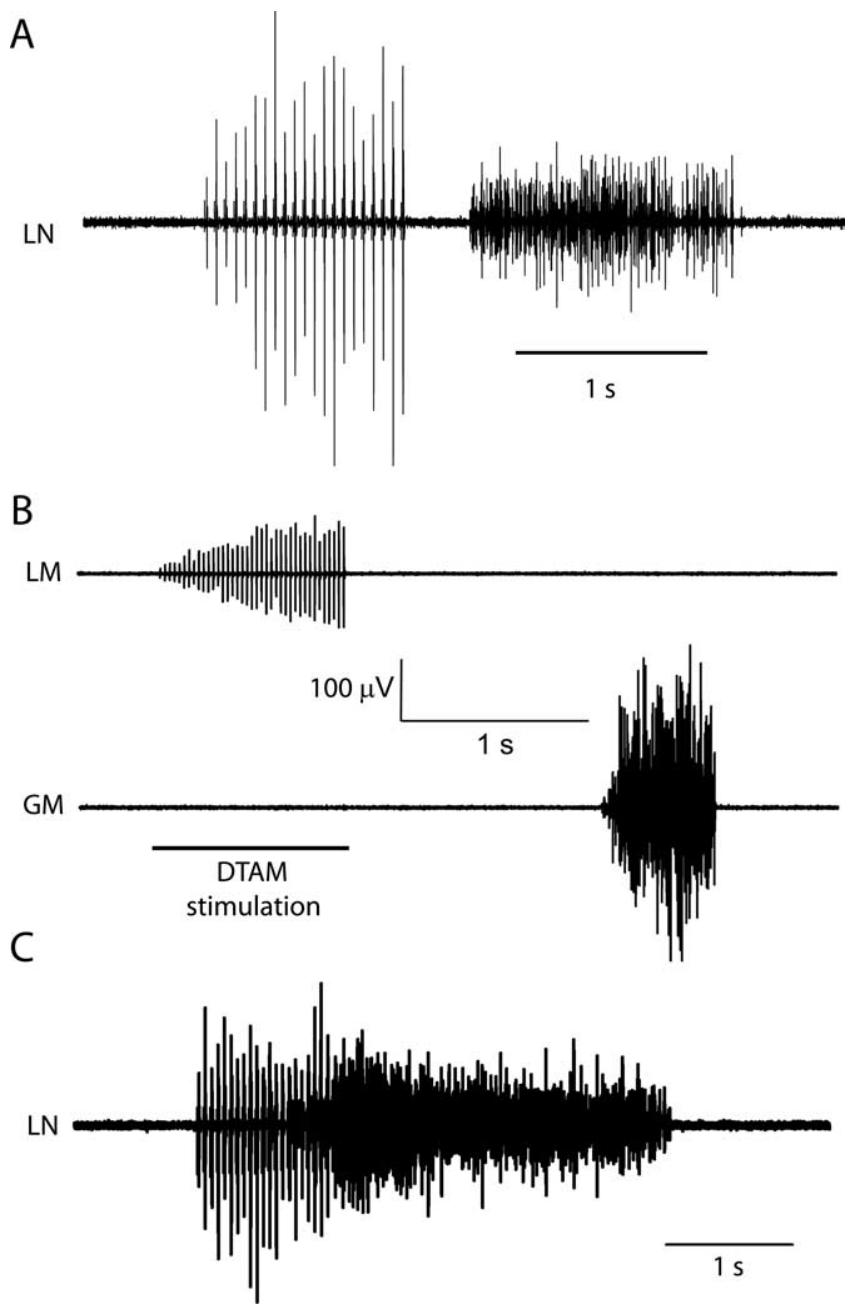

Figure 8. DTAM stimulation induces glottal bursts. $A$, After stimulation in DTAM, highfrequency unpatterned bursts of activity, recorded from N.IX-X, often follow laryngealmuscle-associated CAPs. The durations of these unpatterned bursts are similar to spontaneous glottal bursts (Fig. 2A,B). B, EMG recordings from laryngeal (top trace) and glottal (bottom trace) muscles. Top trace shows stimulus-locked CAPs in laryngeal dilator muscle (LM) but not glottal muscle (GM); poststimulation activity is only present in glottal muscle. C, The glottal burst delay after DTAM stimulation requires GABA transmission; bath application of bicuculline $(1 \mu \mathrm{m})$ eliminates or drastically reduces this delay $(n=5)$. LN, Laryngeal nerve.

nearly all tetrapod vocalizations are coupled. Ancestors of Xenopus were terrestrial anurans (Trueb, 1996) and, although Xenopus diverged from these frogs $\sim 150$ million years ago (Evans et al., 2004), their hindbrain vocal networks share a common origin. In Rana pipiens, a terrestrial anuran, calls made during expiration consist of trills caused by the repeated opening and closing of the glottis (Schmidt, 1972). Schmidt (1992) recorded DTAM activity correlated with fictive vocalizations, and lesions suggested that vocal pattern generation required the cooperative action of n.IX-X and DTAM. A robust reciprocal connection also exists between DTAM and n.IX-X in Xenopus, suggesting that a cooperative mechanism of vocal production operates in aquatic as well as terrestrial frogs.

Despite similarities between Rana and Xenopus, the fact that Xenopus sings without respiration mandates major changes to the vocal circuit. In terrestrial frogs, glottal opening allows air to move across the vocal folds to produce sound. Xenopus, conversely, possesses a sound-producing mechanism that is independent of air movement and therefore does not require glottal

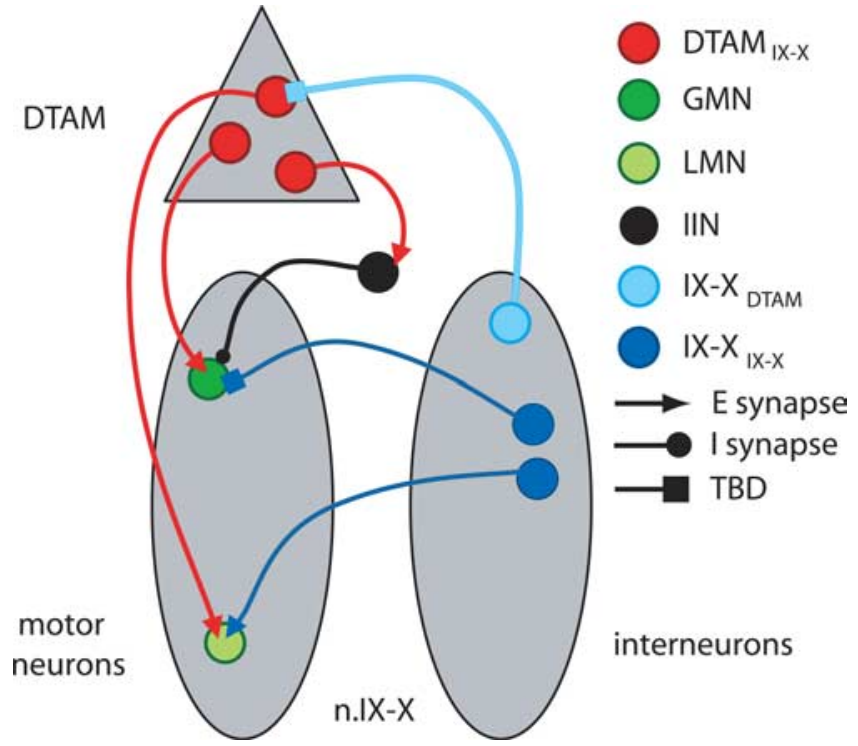

Figure 9. Updated model of the Xenopus laevis vocal-respiratory hindbrain circuit. Graphical representations of DTAM (gray triangle, shown unilaterally) and n.IX-X (gray ovals, shown bilaterally). For clarity, only motor neurons are shown in the left $n . I X-X$, and only interneurons are illustrated in right n.IX-X; in reality, all neuronal pools exist bilaterally. Motor and premotor neurons of the Xenopus vocal-respiratory circuit have been described previously (Zornik and Kelley, 2007). Here, we have shown that DTAM ${ }_{1 X-x}$ neurons provide excitatory inputs to both LMNs and glottal motor neurons (GMNs). Our data also indicate the existence of an inhibitory interneuron (IIN; black) that is activated by DTAM; its location (likely in either n.IX-X or DTAM) is as yet uncertain. Another study showed that commissural IX-X $X_{I X-X}$ neurons also provide excitatory (APMA receptor mediated) inputs onto LMNs (Zornik, 2006), suggesting that LMNs can be activated by at least two premotor neuron pools. The valence and transmitters of other neurons in the circuit (including IX- $X_{\text {DTAM }}$ neurons and IX- $X_{I X-X}$ neurons targeting glottal motor neurons) remain to be determined. E, Excitatory; I, inhibitory; TBD, to be determined.

opening. A clue to how this change might have occurred lies in the observation that DTAM stimulation during glottal bursts causes short pauses in activity; during stimulation, glottal motor neurons receive hyperpolarizing potentials. Thus, stimulating DTAM during a glottal burst may trigger inhibitory inputs, which briefly overpower excitatory premotor respiratory inputs, accounting for the gaps in activity. One possibility is that an excitatory connection between DTAM and glottal motor neurons in terrestrial frogs changed to an inhibitory one, either directly or via an inhibitory interneuron. If DTAM is homologous to the PB, then its role in terrestrial frogs may in part have been to synchronize expiration with calling and may thus be the site for evolutionary changes in Xenopus that led to the uncoupling of breathing and calling.

\section{References}

Bass AH, Baker R (1997) Phenotypic specification of hindbrain rhombomeres and the origins of rhythmic circuits in vertebrates. Brain Behav Evol 50 [Suppl 1]:3-16.

Brahic CJ, Kelley DB (2003) Vocal circuitry in Xenopus laevis: telencephalon to laryngeal motor neurons. J Comp Neurol 464:115-130.

Doyle MW, Bailey TW, Jin YH, Andresen MC (2002) Vanilloid receptors presynaptically modulate cranial visceral afferent synaptic transmission in nucleus tractus solitarius. J Neurosci 22:8222-8229.

Evans BJ, Kelley DB, Tinsley RC, Melnick DJ, Cannatella DC (2004) A mitochondrial DNA phylogeny of African clawed frogs: phylogeography and implications for polyploid evolution. Mol Phylogenet Evol 33:197-213.

Feldmeyer D, Lubke J, Silver RA, Sakmann B (2002) Synaptic connections between layer 4 spiny neurone-layer $2 / 3$ pyramidal cell pairs in juvenile rat barrel cortex: physiology and anatomy of interlaminar signalling within a cortical column. J Physiol (Lond) 538:803-822.

Hollis DM, Boyd SK (2005) Distribution of GABA-like immunoreactive cell 
bodies in the brains of two amphibians, Rana catesbeiana and Xenopus laevis. Brain Behav Evol 65:127-142.

Jürgens U (2002) Neural pathways underlying vocal control. Neurosci Biobehav Rev 26:235-258.

Kelley DB (1980) Auditory and vocal nuclei in the frog brain concentrate sex hormones. Science 207:553-555.

Kelley DB, Tobias ML (1999) The vocal repertoire of Xenopus laevis. In: The design of animal communication (Hauser M, Konishi M, eds), Cambridge, MA: MIT.

Luksch H, Walkowiak W, Munoz A, ten Donkelaar HJ (1996) The use of in vitro preparations of the isolated amphibian central nervous system in neuroanatomy and electrophysiology. J Neurosci Methods 70:91-102.

Markram H, Lubke J, Frotscher M, Roth A, Sakmann B (1997) Physiology and anatomy of synaptic connections between thick tufted pyramidal neurons in the developing rat neocortex. J Physiol (Lond) 500:409-440.

Rhodes HJ, Yu HJ, Yamaguchi A (2007) Xenopus vocalizations are controlled by a sexually differentiated hindbrain central pattern generator. J Neurosci 27:1485-1497.

Schmidt RS (1972) Action of intrinsic laryngeal muscles during release calling in leopard frog. J Exp Zool 181:233-243.

Schmidt RS (1992) Neural correlates of frog calling: production by two semi independent generators. Behav Brain Res 50:17-30.

Simpson HB, Tobias ML, Kelley DB (1986) Origin and identification of fibers in the cranial nerve IX-X complex of Xenopus laevis: Lucifer Yellow backfills in vitro. J Comp Neurol 244:430-444.

Smotherman M, Kobayasi K, Ma J, Zhang S, Metzner W (2006) A mechanism for vocal-respiratory coupling in the mammalian parabrachial nucleus. J Neurosci 26:4860-4869.

Tobias ML, Viswanathan SS, Kelley DB (1998) Rapping, a female receptive call, initiates male-female duets in the South African clawed frog. Proc Natl Acad Sci USA 95:1870-1875.

Tobias ML, Barnard C, O’Hagan R, Horng S, Rand M, Kelley DB (2004) Vocal communication between male Xenopus laevis. Anim Behav 67:353-365.

Trueb L (1996) Historical constraints and morphological novelties in the evolution of the skeletal system of pipid frogs (Anura: Pipidae). Oxford: Clarendon.

Vignal C, Kelley D (2007) Significance of temporal and spectral acoustic cues for sexual recognition in Xenopus laevis. Proc Biol Sci 274:479-488.

Wetzel DM, Kelley DB (1983) Androgen and gonadotropin effects on male mate calls in South African clawed frogs, Xenopus laevis. Horm Behav 17:388-404.

Wetzel DM, Haerter UL, Kelley DB (1985) A proposed neural pathway for vocalization in South African clawed frogs, Xenopus laevis. J Comp Physiol [A] 157:749-761.

Wierenga CJ, Wadman WJ (2003) Functional relation between interneuron input and population activity in the rat hippocampal cornu ammonis 1 area. Neuroscience 118:1129-1139.

Yager D (1992) A unique sound production mechanism in the pipid anuran Xenopus. Zool J Linn Soc 104:1559-1567.

Yamaguchi A, Kelley DB (2000) Generating sexually differentiated vocal patterns: laryngeal nerve and EMG recordings from vocalizing male and female African clawed frogs (Xenopus laevis). J Neurosci 20:1559-1567.

Zornik E (2006) Regulating breathing and calling in an aquatic frog: neuronal networks in the Xenopus laevis hindbrain. $\mathrm{PhD}$ thesis, Columbia University.

Zornik E, Kelley DB (2007) Breathing and calling: neuronal networks in the Xenopus laevis hindbrain. J Comp Neurol 501:303-315. 